certainty' dimension, so that prevalent ideations, and thus 'normal' conditions, can also be accommodated (Marazziti et al, 1999). Thus, insight can be considered to be intertwined with several other dimensions, yet to be identified, and can become disturbed when these other dimensions are altered. In our opinion, therefore, insight is a phenomenon that is only apparently heterogenous, and in fact is strictly related to other variables and/or clinical core features, so that it may well respond to serotonergic drugs and behavioural therapy, as underlined by O'Dwyer \& Marks. The response of insight to various drugs may depend on how close are the links with the other dimension primarily disturbed (e.g. affect; certainty/uncertainty; anxiety). Naturally, these considerations demand new operational criteria that should complete, if not replace, current diagnostic criteria.

Insel, T. R. \& Akiskal, H. S. (1986) Obsessivecompulsive disorder with psychotic features: a phenomenological analysis. American Journal of Psychiatry, 143, 1527-1533.

Marazziti, D., Akiskal, H. S., Rossi, A., et al (1999) Alteration of the platelet serotonin transporter in romantic love. Psychological Medicine, 29, 74I-745.

O'Dwyer, A.-M. \& Marks, I. (2000) Obsessivecompulsive disorder and delusions revisited. British Journal of Psychiatry, I76, 28I-284.

D. Marazziti Dipartimento di Psychiatria Neurobiologia, Farmacologia e Biotecnologie, University of Pisa, via Roma, 67, 56100 Pisa, Italy

\section{Practical application of structured risk assessment}

I was disappointed by Szmukler's (2001) negative response to Dolan \& Doyle's (2000) excellent review of attempts to measure the risk of violence in psychiatric patients. His pessimism about the practical application of structured risk assessment results from a misunderstanding of the way in which these instruments may be used. First, he emphasises the low baseline. Of course, we do not know the baseline, as the information has never been collected accurately in this country. The $6 \%$ in 6 months cited in the letter derives from retrospective ratings by clinicians. Studies in the USA show that rates of violence by psychiatric patients may rise threefold if self-report is supplemented by official records and by the account of a key informant. In any case, the low baseline is irrelevant. Most risk assessment tools were developed on high-risk populations, usually people who had already committed serious offences. They were not designed to be applied to all patients. If these instruments were applied to those $6 \%$ of patients with a record of recent violence, would they assist future management by identifying those who were at highest risk of a repetition? I do not know the answer, but it is a sensible and important question.

The second problem is an unrealistic expectation of what such instruments can do. They are a supplement to good clinical practice, not a substitute for it. In many parts of Canada, for example, the Violence Risk Appraisal Guide (Harris et al, 1993) is administered to all patients admitted to a medium secure unit. The results do not dictate future management but, like the results of any other investigation, they inform it. I have no good answer to the question of why we are not evaluating this practice in the UK. When one concentrates on small, high-risk populations, the resource arguments lose this force. With medium secure admissions in this country averaging $>18$ months in duration, at $£ 100000$ per patient per annum, a few hours of a psychologist's time is neither here nor there.

It is unfortunate that British psychiatry has been slow to look at the application of these instruments, on which much basic research has already been done. The situation is even more depressing because National Health Service Trusts, fearful of lawsuits, are insisting on the introduction of standardised risk assessments. In most cases, they are not of proven value. Trusts in different areas use different instruments, making evaluation more difficult. The measures are likely to be employed indiscriminately, becoming just another form to be filled in. Would it not be better if this initiative were driven by clinicians, and underpinned by a sound methodology for evaluation?

Psychiatry must not persist in assuming that violence, an uncommon complication of mental disorder, is unimportant because of its rarity. Reforming the Mental Health Act (Department of Health, 2001) illustrates that concern about violence dominates the thinking of politicians in this area. It is unlikely that they are going to lose votes by overstating the level of risk associated with psychiatric patients, so the profession is going to have to come up with something better than bland reassurance. We are in a weak position, so long as we lag behind North America and parts of Europe, both in our use of existing risk-assessment technology, and in research into violence. The paper by Dolan \& Doyle should stimulate us to make up some of that lost ground.

Department of Health (200I) Reforming the Mental Health Act. Cm 5016. London: Stationery Office.

Dolan, M. \& Doyle, M. (2000) Violence risk prediction. Clinical and actuarial measures and the role of the Psychopathy Checklist. British Journal of Psychiatry, I77, 303-3II.

Harris, G. T., Rice, M. E. \& Quinsey, V. L. (1993) Violent recidivism of mentally disordered offenders: the development of a statistical prediction instrument. Criminal Justice and Behaviour, 20, 315-335.

Szmukler, G. (200I) Violence risk prediction in practice (letter). British Journal of Psychiatry, 178, 84-85.

A. Maden Academic Centre, Trust Headquarters, EHF NHS Trust, Uxbridge Road, Southall, Middlesex UBI 3EU

\section{Classic text still accessible}

Professor Goldberg's (2001) excellent retrospective demonstrates the breadth of reading that used to be part of psychiatric training. His statement that General Psychopathology by Karl Jaspers is "out of print" is happily not the case. It was republished by Johns Hopkins University Press in 1997 in two volumes and is readily available via internet booksellers.

As Goldberg states, the work influenced a whole generation of psychiatrists and remains a very readable text, full of detail and close observation, which harks back to a time when meticulous detail and careful attention to language were essentials of good psychiatric practice. Any student of psychiatry, at any level, would do well to take a look.

Goldberg, D. (2001) Ten Books. Chosen by David Goldberg. British Journal of Psychiatry, 178, 88-91.

Jaspers, K. (1997) General Psychopathology, Vols I \& 2 (transl. J. Hoenig \& M.W. Hamilton). Baltimore, MD: Johns Hopkins University Press.

A. J.Warren Cygnet Hospital, Ealing,

London W5 2 HT 